\title{
On the Numerical Solution of a Differential-Difference Equation Arising in Analytic Number Theory
}

\author{
By J. van de Lune and E. Wattel
}

1. Abstract and Introduction. In the January 1962 issue of this journal R. Bellman and B. Kotkin published a short paper under the same title as this one (cf. [1]). In that paper Bellman and Kotkin presented some of their results concerning the numerical computation of the continuous function $y(x)$, defined by

$$
\begin{aligned}
& y(x)=1 \quad(0 \leqq x \leqq 1) \\
& y^{\prime}(x)=-\frac{1}{x} \cdot y(x-1) \quad(x>1) .
\end{aligned}
$$

Tables of $y(x)$ were given for $x=1(0.0625) 6$ and $x=6(1) 20$. In the process of extending these tables beyond $x=20$ we discovered that the second table was rather inaccurate for all values of $x>9$. Bellman and Kotkin found, for example, that $y(20)=0.149 \cdot 10^{-8}$, whereas the actual value of $y(20)$ can be shown to be smaller than $10^{-20}$. Moreover, in view of the method used by Bellman and Kotkin, one may expect that it would be quite time consuming to compute $y(x)$ for values of $x$ up to say $x=1,000$.

In this paper we describe a different method which enables us to compute $y(x)$ for values of $x$ up to about "as far as one would like."

2. The Main Formula and Some of its Consequences. The function $y(x)$ defined in the introduction satisfies the following fundamental

LEMMA 1.

$$
x \cdot y(x)=\int_{x-1}^{x} y(t) d t \quad(x \geqq 1) .
$$

Proof. Cf. de Bruijn [2].

A simple consequence of this lemma is

LEMma 2.

$$
y(x)>0 \quad(x \geqq 0) .
$$

As an easy consequence of this lemma and the definition of $y(x)$ we find that $y(x)$ is monotonically decreasing on $x \geqq 1$.

Lemma 3. $y(x)$ is concave on $x \geqq 1$.

Proof. From the definition of $y(x)$ it follows that

so that

$$
y(x)=1-\log x \quad(1 \leqq x \leqq 2),
$$

$$
y(x) \text { is concave on } 1 \leqq x \leqq 2 .
$$

Received June 23, 1968, revised October 1, 1968. 
Also from the definition of $y(x)$ it is easily seen that $y(x)$ is twice differentiable on $x>2$, whereas $y(x)$ is precisely once differentiable at $x=2$. On $x>2$ we have

$$
y^{\prime \prime}(x)=\frac{d}{d x}\left(-\frac{1}{x} \cdot y(x-1)\right)=\frac{1}{x^{2}} \cdot y(x-1)+\frac{-1}{x} \cdot \frac{-1}{x-1} \cdot y(x-2)>0 .
$$

Since $y(x)$ is concave on the intervals $1 \leqq x \leqq 2$ and $x>2$ and differentiable at $x=2$, we may conclude that $y(x)$ is concave on $x \geqq 1$.

Lemma 4.

$$
y(x)<\frac{1}{2 x-1} \cdot y(x-1), \quad(x \geqq 2) .
$$

Proof. On $x \geqq 2$ we have by Lemma 3 that

$$
x \cdot y(x)=\int_{x-1}^{x} y(t) d t<\frac{1}{2}\{y(x-1)+y(x)\}
$$

and consequently

$$
y(x)<\frac{1}{2 x-1} \cdot y(x-1) .
$$

From Lemma 4 one easily deduces by induction that

$$
y(n)<\frac{1}{3 \cdot 5 \cdot 7 \cdot \cdots(2 n-1)}=\frac{2^{n} \cdot n !}{(2 n) !}, \quad(n=2,3,4, \cdots) .
$$

Hence, for example,

$$
y(20)<\frac{2^{20} \cdot 20 !}{40 !}=\frac{2^{20}}{21 \cdot 22 \cdot 23 \cdot \cdots 40}<\frac{2^{20}}{20^{20}}=10^{-20} .
$$

This rough upper bound for $y(20)$ shows that the value of $y(20)$ given by Bellman and Kotkin is not even of the proper order.

3. The Numerical Computation of $y(x)$. Our starting point is the result of Lemma 1

$$
\begin{gathered}
y(x)=1 \quad(0 \leqq x \leqq 1), \\
(x+1) \cdot y(x+1)=\int_{x}^{x+1} y(t) d t \quad(x \geqq 0) .
\end{gathered}
$$

We have already mentioned that

$$
y(x)=1-\log x, \quad(1 \leqq x \leqq 2)
$$

so that we only have to compute $y(x)$ on $x>2$.

If we approximate the integral

$$
I=\int_{x_{0}}^{x_{0}+1} y(t) d t, \quad\left(x_{0} \geqq 1\right)
$$

by means of the trapezoidal formula 


$$
\frac{1}{2 n}\left\{y\left(x_{0}\right)+2 \sum_{k=1}^{n-1} y\left(x_{0}+\frac{k}{n}\right)+y\left(x_{0}+1\right)\right\}
$$

we obtain, because of the concavity of $y(x)$ on $x \geqq 1$, that

$\left(x_{0}+1\right) y\left(x_{0}+1\right)=\int_{x_{0}}^{x_{0}+1} y(t) d t<\frac{1}{2 n}\left\{y\left(x_{0}\right)+2 \sum_{k=1}^{n-1} y\left(x_{0}+\frac{k}{n}\right)+y\left(x_{0}+1\right)\right\}$.

It follows that

$$
y\left(x_{0}+1\right)<\frac{1}{2 n\left(x_{0}+1\right)-1}\left\{y\left(x_{0}\right)+2 \sum_{k=1}^{n-1} y\left(x_{0}+\frac{k}{n}\right)\right\} .
$$

Thus, if one has upper bounds for $y(x)$ at the points

$$
x_{0}+k / n, \quad(k=0,1,2, \cdots, n-1),
$$

one may compute an upper bound for $y\left(x_{0}+1\right)$.

Continuing in this way one may compute upper bounds for $y(x)$ at the points $x_{0}+1+v / n,(v=1,2,3, \cdots)$.

On the other hand, approximating $I$ by

$$
\frac{1}{n} \sum_{k=1}^{n} y\left(x_{0}+\frac{2 k-1}{2 n}\right)
$$

one finds, also because of the concavity of $y(x)$ on $x \geqq 1$, that

$$
y\left(x_{0}+1\right)>\frac{1}{n\left(x_{0}+1\right)} \sum_{k=1}^{n} y\left(x_{0}+\frac{2 k-1}{2 n}\right) .
$$

Hence, as soon as one has lower bounds for $y(x)$ at the points $x_{0}+(2 k-1) / 2 n$, $(k=1,2,3, \cdots, n)$ one may compute a lower bound for $y\left(x_{0}+1\right)$. If one also knows lower bounds for $y(x)$ at the points $x_{0}+k / n,(k=1,2,3, \cdots, n-1)$, one can apply the same method to compute a lower bound for $y\left(x_{0}+1+1 / 2 n\right)$. Repeating this process one finds lower bounds for $y(x)$ at the points $x_{0}+1+k / 2 n$, $(k=2,3,4, \cdots)$. As a starting point for the computations one may take of course $x_{0}=1$.

If one chooses the grid sizes in the above integral-approximating procedures small enough, one may expect that the corresponding upper and lower bounds for $y(x)$ will not differ very much. Actual computations show that this is indeed the case.

Performing the computations on the Electrologica X8 of the Mathematical Centre in Amsterdam, using an ALGOL-60 program (with grid size 0.005), we found that the corresponding upper and lower bounds for $y(x)$ were equal up to at least the first significant digit for all $x<100$.

Using more refined integral-approximating formulae and smaller grid sizes we were able to compute $y(x)$ for values of $x$ up to at least $x=1,000$. Below we include a table for $y(x)$ with an accuracy of five or more significant figures.

Finally we will compare some of the results of Table 1 with the known asymptotic formula of de Bruijn (cf. [2])

$$
y(x) \sim \frac{e^{\gamma}}{(2 \pi x)^{1 / 2}} \exp \left\{1-e^{\xi}+\int_{0}^{\xi} \frac{e^{s}-1}{s} d s\right\} \quad(x \rightarrow \infty),
$$


where $\xi$ is the positive root of $e^{\xi}-1=x \xi$ and $\gamma$ is Euler's constant. De Bruijn's formula can be rewritten in terms of the exponential integral $\operatorname{Ei}(t)=\int_{-\infty}^{t}\left(e^{s} / s\right) d s$ as

$$
y(x) \sim \frac{1}{(2 \pi x)^{1 / 2}} \cdot \frac{1}{\xi} \cdot \exp \{-x \cdot \xi+\operatorname{Ei}(\xi)\}, \quad(x \rightarrow \infty)
$$

which is somewhat more convenient for numerical computations. Writing $B(x)$ for de Bruijn's asymptotic approximation we have

TABLE 1. $y(x)=a(x) \cdot 10^{-b(x)}$

\begin{tabular}{|c|c|c|c|c|c|c|c|c|}
\hline$x$ & $a(x)$ & $b(x)$ & $x$ & $a(x)$ & $b(x)$ & $x$ & $a(x)$ & $b(x)$ \\
\hline 2 & 0.306852 & 0 & 36 & 0.121869 & 62 & 70 & 0.702809 & 147 \\
\hline 3 & 0.486083 & 1 & 37 & 0.622168 & 65 & 71 & 0.162933 & 149 \\
\hline 4 & 0.491092 & 2 & 38 & 0.307395 & 67 & 72 & 0.371471 & 152 \\
\hline 5 & 0.354724 & 3 & 39 & 0.147112 & 69 & 73 & 0.833076 & 155 \\
\hline 6 & 0.196496 & 4 & 40 & 0.682549 & 72 & 74 & 0.183819 & 157 \\
\hline 7 & 0.874566 & 6 & 41 & 0.307253 & 74 & 75 & 0.399153 & 160 \\
\hline 8 & 0.323206 & 7 & 42 & 0.134297 & 76 & 76 & 0.853156 & 163 \\
\hline 9 & 0.101624 & 8 & 43 & 0.570381 & 79 & 77 & 0.179535 & 165 \\
\hline 10 & 0.277017 & 10 & 44 & 0.235551 & 81 & 78 & 72043 & 168 \\
\hline 11 & 0.664480 & 12 & 45 & 0.946492 & 84 & 79 & 0.759361 & 171 \\
\hline 12 & 0.141971 & 13 & 46 & 0.370280 & 86 & 80 & 0.152686 & 173 \\
\hline 13 & 0.272918 & 15 & 47 & 0.141120 & 88 & 81 & 0.302503 & 176 \\
\hline 14 & 0.476063 & 17 & 48 & 0.524252 & 91 & 82 & 0.590640 & 179 \\
\hline 15 & 0.758990 & 19 & 49 & 0.189943 & 93 & 83 & 0.113672 & 181 \\
\hline 16 & 0.111291 & 20 & 50 & 0.671533 & 96 & 84 & 0.215679 & 184 \\
\hline 17 & 0.150907 & 22 & 51 & 0.231788 & 98 & 85 & 0.403511 & 187 \\
\hline 18 & 0.190135 & 24 & 52 & 0.781464 & 101 & 86 & 0.744510 & 190 \\
\hline 19 & 0.223542 & 26 & 53 & 0.257465 & 103 & 87 & 0.135495 & 192 \\
\hline 20 & 0.246178 & 28 & 54 & 0.829313 & 106 & 88 & 0.243271 & 195 \\
\hline 21 & 0.254805 & 30 & 55 & 0.261272 & 108 & 89 & 0.430958 & 198 \\
\hline 22 & 0.248638 & 32 & 56 & 0.805427 & 111 & 90 & 0.753402 & 201 \\
\hline 23 & 0.229371 & 34 & 57 & 0.243046 & 113 & 91 & 0.129996 & 203 \\
\hline 24 & 0.200549 & 36 & 58 & 0.718206 & 116 & 92 & 0.221416 & 206 \\
\hline 25 & 0.166580 & 38 & 59 & 0.207907 & 118 & 93 & 0.372331 & 209 \\
\hline 26 & 0.131725 & 40 & 60 & 0.589802 & 121 & 94 & 0.618228 & 212 \\
\hline 27 & 0.993606 & 43 & 61 & 0.164025 & 123 & 95 & 0.101374 & 214 \\
\hline 28 & 0.716213 & 45 & 62 & 0.447329 & 126 & 96 & 0.164183 & 217 \\
\hline 29 & 0.494179 & 47 & 63 & 0.119673 & 128 & 97 & 0.262667 & 220 \\
\hline 30 & 0.326904 & 49 & 64 & 0.314165 & 131 & 98 & 0.415161 & 223 \\
\hline 31 & 0.207626 & 51 & 65 & 0.809545 & 134 & 99 & 0.648360 & 226 \\
\hline 32 & 0.126782 & 53 & 66 & 0.204821 & 136 & 100 & 0.100059 & 228 \\
\hline 33 & 0.745257 & 56 & 67 & 0.508958 & 139 & 200 & 0.983383 & 530 \\
\hline 34 & 0.422222 & 58 & 68 & 0.124246 & 141 & 500 & 0.505734 & 1558 \\
\hline 35 & 0.230808 & 60 & 69 & 0.298056 & 144 & 1000 & 0.458767 & 3463 \\
\hline
\end{tabular}

TABle 2

\begin{tabular}{rlll}
\hline \multicolumn{1}{c}{$x$} & \multicolumn{1}{c}{$y(x)$} & \multicolumn{1}{c}{$B(x)$} & $\frac{y(x)}{B(x)}$ \\
\hline 20 & $0.246178 \cdot 10^{-28}$ & $0.219619 \cdot 10^{-28}$ & 1.121 \\
100 & $0.100059 \cdot 10^{-228}$ & $0.090892 \cdot 10^{-228}$ & 1.101 \\
1000 & $0.458767 \cdot 10^{-3463}$ & $0.422946 \cdot 10^{-3463}$ & 1.085 \\
\hline
\end{tabular}


Acknowledgement. The authors wish to thank Professor A. van Wijngaarden for his helpful suggestions concerning the numerical computations.

Mathematical Centre

Amsterdam, The Netherlands

1. R. Bellman \& B. Kotkin, "On the numerical solution of a differential-difference equation arising in analytic number theory," Math. Comp., v. 16, 1962, pp. 473-475. MR 26 \#5756.

2. N. G. DE BRUIJN, "The asymptotic behaviour of a function occurring in the theory of primes," J. Indian Math. Soc., v. 15, 1951, pp. 25-32. MR 13, 326.

For an extensive list of literature concerning the function $y(x)$ we refer to

3. J. van De Lune \& E. WatTel, On the Frequency of Natural Numbers $m$ whose Prime Divisors are all Smaller than $m^{\alpha}$, Mathematical Centre, Amsterdam, Report ZW 1968-007, 1968. 